\title{
Sharing Manipulated Heart Rate Feedback in Collaborative Virtual Environments
}

\author{
Arindam Dey* \\ University of Queensland
}

\author{
Hao Chen ${ }^{\dagger}$ \\ University of Canterbury
}

\author{
Ashkan Hayati \\ University of South Australia
}

Robert W. Lindeman ${ }^{\text {II }}$

University of Canterbury

\author{
Mark Billinghurst ${ }^{\S}$ \\ University of South Australia
}

\begin{abstract}
We have explored the effects of sharing manipulated heart rate feedback in collaborative virtual environments. In our study, we created two types of different virtual environments (active and passive) with different levels of interactions and provided three levels of manipulated heart rate feedback (decreased, unchanged, and increased). We measured the effects of manipulated feedback on Social Presence, affect, physical heart rate, and overall experience. We noticed a significant effect of the manipulated heart rate feedback in affecting scariness and nervousness. The perception of the collaborator's valance and arousal was also affected where increased heart rate feedback perceived as a higher valance and lower arousal. Increased heart rate feedback decreased the real heart rate. The type of virtual environments had a significant effect on social presence, heart rate, and affect where the active environment had better performances across these measurements. We discuss the implications of this and directions for future research.
\end{abstract}

\section{INTRODUCTION}

This paper explores how manipulating physiological feedback can change the emotional experience for people in a shared Virtual Environment (VE). Previous research has shown that immersive Virtual Reality (VR) is capable of creating strong emotional responses. For example University of North Carolina's famous Pit Experiment uses VR to create the illusion that people are standing on a plank over a two storey pit, and they report that many users exhibit a classic fear response [13]. Seinfield et al. [19] created a more recent variant, where people rode an open elevator up the side of an extremely tall virtual building, and felt increasing anxiety and fear as the height increased. The entire VR gaming industry is based on how VR can provide excitement and create emotional experiences, and there are many other examples. Understanding how emotions are experienced in VR could lead to novel applications beyond gaming, such as therapy and rehabilitation.

One way that emotional response to VR can be measured is through physiological sensing. In the Pit Experiment [13] the user's heart rate, skin conductance, and skin temperature was measured, and was shown to vary dramatically as the user entered the virtual pit room. There are several other examples of the use of physiological signals in VR as detailed in Section 2. There is another line of research where feedback of these physiological signals were provided in order to enhance the experience of VEs. For example, Dey et al. [7] found that physiological cues, such as heart rate, can be shared in a collaborative VR environment to enhance the user experience.

\footnotetext{
*e-mail: a.dey@uq.edu.au

†e-mail:hao.chen@pg.canterbury.ac.nz

†e-mail:faray030@mymail.unisa.edu.au

\$email: mark.billinghurst@unisa.edu.au

Itemail:gogo@hitlabnz.org
}

In this case graphical and audio cues were used to convey the heart rate of a game player in VR to an observer also in VR. The cues used were mapped one to one with the VR player's actual heart rate, and they found by sharing them they were able to make the observer more excited as the excitement of the player in VR increased.

However, there is no need for the audio and visual cues for the user's heart rate to map exactly to the player's actual heart rate. This work extend Dey's work and explores what happens when one user is hearing a manipulated heartrate cue of another user in the same VR experiences. For example, what happens when the player's heartrate is normal, but the audio and visual cues send to the observer is from a sped up version of the player's actual heartrate? This is an interesting question because if the observer's experience in VR can be enhanced through manipulation of a player's physiological cues, this could be used as a technique to create a better experience. Dey et al. [5] explored this question in a single user setup and found heart rate manipulation can manipulate certain emotions when experiencing VEs.

\subsection{Novelty and Contribution}

Although several researchers have explored how physiological cues can be shared in collaborative VR experiences, this is the first time that heart rate manipulation has been explored. So the main novelty of this paper is that, for the first time, the effect of manipulated heart rate feedback on the perception of collaborator's and self's emotional state is evaluated. This is important because by understanding this effect, we can manipulate such physiological feedback to create social connection and empathy between the collaborators, which may lead to better task performance. There are two main contributions of the paper. First, we presented a collaborative system that shares heart rate feedback between collaborators in real-time using multi-sensory channels. Second, we have thoroughly studied, using a series of qualitative and quantitative measurements, the effects that heart rate manipulation can have on emotional responses in a second user in shared VR experiences.

The rest of the paper is organized as follows. We first review related work in measuring and sharing physiological cues in VR (Section 2). In Section 3, we describe our experimental system and how we developed for manipulating the representation of heart rate signals in VR. Next we present our user study design (Section 4) and results from the study (Section 5). In Section 6, we discuss the results in relation to the hypotheses. Finally we end the paper with some conclusions and directions for future research in Section 7.

\section{Related Work}

In our work, we are investigating the effects of heart rate feedback manipulation on emotions and social presence in collaborative VR environments. In the last few years, researchers began using VR environments as a tool to investigate emotions. Felnhofer et al. [9] reported that VEs accurately triggered emotions in five different kinds of virtual park scenarios, as measured by emotional arousal 
and presence using galvanic skin response (GSR). Riva et al. [16] argued that the degree of presence experienced in a VE has a strong influence on the experienced emotional states. Emotional arousal has been measured and used in different military applications. For example, Rizzo et al. [17] created emotional states for military training in a post-war scenario and Roy et al. [18] used emotion elicitation in VR to treat post traumatic stress disorder (PTSD). In other work, Wiederhold et al. [26] reported treating people with flying phobia using VR and measured physiological measures such as heart rate and skin conductance.

There is another, smaller group of work where besides just measuring emotions and/or physiological data, researchers have investigated ways to visualize and communicate that data. For example, sharing physiological data in a collaborative video conferencing was investigated by Tan et al. [23], and found physiological data sharing creates a higher sense of co-presence than audio only communication without physiological data sharing. Fukushima et.al [10] found that when participants were watching movies clips and at the same time they could hear their own heart beat, they were confused about hearing their own heartbeat in the headphone, and after watching for several minutes thought the heart beat was from the people in the movie. Shah et.al [21] discovered that people able to hear their own heart beat are more empathetic. In their study, they asked subjects to count their heart beats without monitoring their pulse and those who had counted their heart beats most accurately were better at answering questions relating to the characters' emotions when they were watching the video clips of social interactions. Slovak et.al [22] investigated the HR sharing visually and aurally in the social context and found HR communication can have strong effects on intimacy between the subjects. Another work from Hassib et.al [12] shows HR sharing helped participants calm down when they were angry and could inform them when their partner was excited about something and then conversations was triggered between them. In their work, they designed a system-HeartChat - which is a mobile chat application with heart rate information embedded into a chat environment. However such reported work in VR is rare.

In a recent work, Dey et al. [7] investigated the effect of sharing real time audio-visual heart rate cues of one player to another in two different (scary and calm) collaborative VR gaming environments. They reported that being able to see the collaborator's heart rate in real time created a trend towards higher positive affect than not seeing it, and that this made the collaborators connect better with each other. In another work from Dey et.al [6], they shared the realtime HR between the participants when participants were playing three types of VR game (jungle safari, escape room, and furniture arrangement) and found HR sharing increased dominance, feeling the presence of the collaborator, and understanding the emotional state of the collaborator. Bernal and Meas [1] recently reported work where they visualized emotions using avatars in VEs. After measuring GSR and heart rate data, they represented emotions visually in two different ways: (1) growing the fur on the skin of an avatar when arousal is high, and (2) intensifying the brightness or colour change to highlight the avatar when arousal is high. In other work, Chen et al. [4] investigated how to provide heart rate feedback to users in a VE using multi-sensory channels, and whether showing the real-time heart rate cues in VEs can make the user more aware of her own emotional state. They reported that participants preferred having sensory feedback, and among combinations of audio, visual, and haptic feedback, participants preferred audio-haptic feedback the most, while visual feedback was found to be distracting. Our current work uses the findings of Chen et al. [4] and provides only audio-haptic feedback. The most closely related work to our current study is reported by Dey et al. [5] where they manipulated the heart rate feedback of the user and provided the feedback in real-time to the user while experiencing VR. They reported that five emotions in particular was influenced by the manipulation of heart rate feedback - interested, excited, scared, nervous, and afraid.

Summary: The current body of knowledge established that physiological signals, including heart rate, are a valid measurement of emotions. Researchers have widely used these measurements in VR to study the emotional affects of both single-user and collaborative VR experiences. However, none of the earlier studies explored the effects of sharing a manipulated heart rate feedback of one collaborator to the other in multi-user VR experiences.

\section{Experimental System Design}

In this user study, we designed six VR scenes. Three of them are passive games, called Safari touring. In these three VR scenes, participants were standing on the back of a virtual pickup, which moved on its own without any intervention from the players. The two participants appeared in the VR scenes as two virtual heads. They could rotate their virtual head by rotating their real head to see each other in their head mounted display (HMD), as shown in the Figure 2f. Each Safari VR experience is about 4 minutes in length. We designed the other three VR scenes to be active, and a zombie shooting game. In these VR scenes, the two participants need work together to shoot zombies and spiders coming at them from four different directions (Figure2c). We used Photon Engineer ${ }^{1}$ to make the Zombie shooting game into a networked game. The two participants could see each other's head and the virtual guns they held in their hands. We used the HTC Vive ${ }^{2}$ HMD, controllers and tracking system to help participants experience the VR scenes.

When two participants were playing the games, they could hear each other's real-time heart beat. We used the Zephyr BTLE BioModule Device ${ }^{3}$ to capture the real-time heart rate (see Figure 1). The sensor was strapped around the participant's chest. To share the heart beat, we set up a server to collect the heart rate data of one participant via Bluetooth Low Energy(BLE) and streamed the data to the computer connected to the other participant's HTC Vive through the UDP protocol. For example, the server collected the heart rate data from the sensor on participant 1 and then streamed the data to PC2. The heart rate signal acquired from the participants was fed into Unity. Within Unity a script analyzed the incoming ECG data and sonified it by playing a pre-selected audio clip at intervals matching the participants' heart rates. The script also have a function to analyze HR and implemented HR to be different vibrating strength on HTC vive controllers according to the HR values.

\section{User Study}

To explore the effects of providing manipulated heart rate feedback in collaborative VEs-where both collaborators will get the feedback of the other collaborator (and not of their own) - we conducted a rigorous within-subjects user study with 24 participants grouped into 12 pairs.

\subsection{Independent Variables}

There were two independent variables in this experimentManipulation Level and Environment Type, as described next. Being a within-subjects study, all participants experienced all levels of both of the independent variables. The presentation order of the independent variables was counterbalanced.

\subsubsection{Manipulation Level $\rightarrow-20 \%, 0 \%,+20 \%$}

In a previous study with non-collaborative tasks, it was found that $\pm 30 \%$ manipulation of heart rate feedback was easily identifiable by the participants, however, $\pm 15 \%$ went unnoticed [5]. Learning from that study and after our own pilot study we identified that manipulation levels of $\pm 20 \%$ was not noticeable by participants.

\footnotetext{
${ }^{1}$ https://www.photonengine.com/

${ }^{2}$ https://www.vive.com/

${ }^{3}$ https://www.zephyranywhere.com
} 


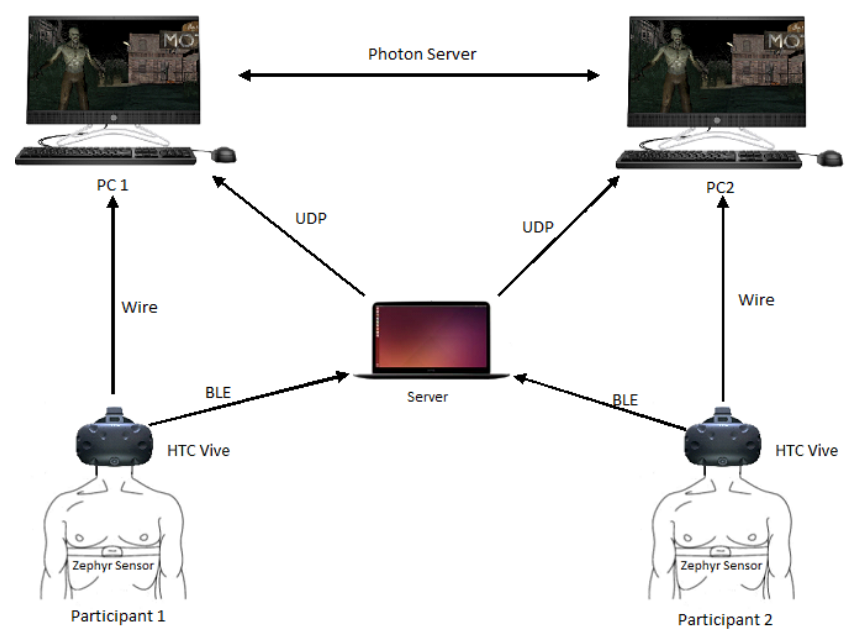

Figure 1: The experimental system

Hence, we had three levels of manipulation $-20 \%, 0 \%$ (real), and $+20 \%$. To collect the real-time heart rate data we used Zephyre ${ }^{4}$ heart rate sensors that participants wore on their chests.

Following the insights from an earlier experiment by Chen et al. [4], all of the heart rate feedback was provided using real-time audio-haptic channels. Haptic feedback was provided using the HTC Vive controllers and the audio feedback was provided using Logitech noise cancelling headphones, this feedback was synchronized to the participants' real heart beats. One important fact to note is that the participants were unaware of the manipulation. They were told that the feedback they were getting was real and in real time. This was done to ensure that the participants were not primed and respond with that knowledge in mind.

\subsubsection{Collaborative Environments $\rightarrow$ Active (shooting) and Passive (safari)}

We designed two different collaborative environments with different levels of interactivity. We were interested to identify what effect do interactions have on the perception of the manipulated feedback? Using the Unity $3 \mathrm{D}$ game engine ${ }^{5}$, we created three similar versions for each of the environments to properly counterbalance with the manipulation levels and avoiding learning effect. All of the VR experiences lasted for four minutes. In both of the environments, we asked participants to talk freely with each other as much as they wished.

\section{Active (shoot to survive):}

In this environment, participants were placed in an abandoned industrial area at night where multiple zombies were attacking them (Figure 2(a-c)). They had two revolvers, one in each hand, to shoot at the zombies to survive. There were two participants (players) shooting at the zombies and if one player died the other would also die, hence they had to help each other and communicate. Zombies could come and attack from any direction, however, when they appeared they were visible from a distance giving enough time to identify and shoot. We supplemented the environment with appropriate sound effects. Players were able to see each other in the environment as a virtual unisex head model. The orientation and location of that model was updated in real time based on the players' movements in the real world. We chose to use a same unisex head model, instead of gender-based avatars, for both participants to avoid any confounding effect of different avatar representations.

\footnotetext{
${ }^{4}$ https://www.zephyranywhere.com

${ }^{5}$ https://unity3d.com/
}

We designed the environment in a way that none of the players die as we wanted all participants to experience the virtual environment for the entire four minutes. However, participants were not aware of the fact. We created three versions of the environment where the location was slightly different but the lighting conditions and number of attacking zombies were the same.

Passive (jungle safari):

The passive experience was based on a jungle safari with various animals moving around in the environment and supplemented with suitable sound effects (Figure 2(d-f)). Both participants were tourists and placed in a standing position on the back of a virtual car moving along without any interaction from the players. However, they could see each other and, if chosen to do so, they could communicate with each other verbally similar to what tourists may do in a real jungle safari. Although this environment did not require any active collaboration to perform a task but it collaborative in a sense that it required both of the participants to explore a leisurely jungle safari together. Most of the visual effects of interest were presented in front of the participants' eyes within a $200^{\circ}$ horizontal field of view. However, there were sound effects that originated behind the participants using spatial sound playback. Similar to the earlier environment a virtual unisex head model was provided to indicate the location of the collaborator.

In the physical world, the both of the participants were standing with a hand-rest in front to maintain balance, if needed. They were allowed to look around and rotate their heads to experience the VE at will. However, they were not allowed to walk as we wanted to avoid any elevated physiological signals due to locomotion.

\subsection{Dependent Variables}

Our main goal of the experiment was to identify the effects of manipulated heart rate feedback on self-perception of emotions and the emotions of the collaborator. We were also interested to identify how the collaboration was affected due to the manipulated feedback. As such we collected subjective data through four validated instruments. First, the Positive and Negative Affect Schedule (PANAS) [25] was used to collect the participant's emotional state and we also asked participants to rate their collaborator's emotional state as also using the same survey. Second, we used Inclusion of the Other in Self (IOS) scale to measure how the participants felt connected to their collaborator. Third, we used the Social Presence Questionnaire (SPQ) [11] to measure the overall Presence felt in the collaborative tasks. We measured participants' general emotions and arousal using self-assessment manikin [2]. Additionally, we asked participants six subjective questions on a five-point Likert scale. As an objective variable we collected the participants' raw heart rate data.

\subsection{Task and Procedure}

The experimental environments were collaborative, so the tasks were done in pairs. In the shooting task, participants had to look for zombies coming from all directions and shoot them to survive. They also had to help the other collaborator so that neither of them die. Overall, this environment required more interaction with the environment and the other collaborator. Hence, we classify this task as an active task. The other task-safari-simply required participants to look around and experience a virtual jungle safari. There was no interaction with the environment beyond just looking around and interaction with the other collaborator was not required. We term this as a passive task. During all of the tasks there was a continuous heart rate feedback of the other player provided. All participants were categorically told that the feedback they were receiving was that of the other collaborators and not their own and that the feedback was in real time.

Each pair of participants had to go through all environments and heart rate manipulation levels. Hence, they had to perform 


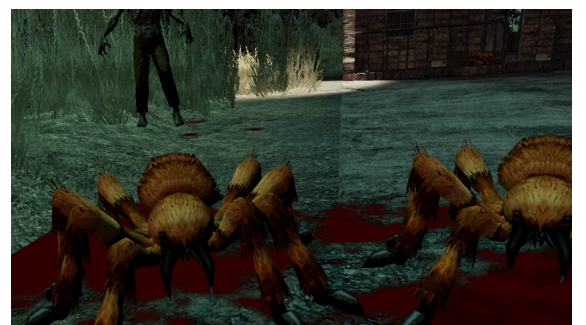

(a)

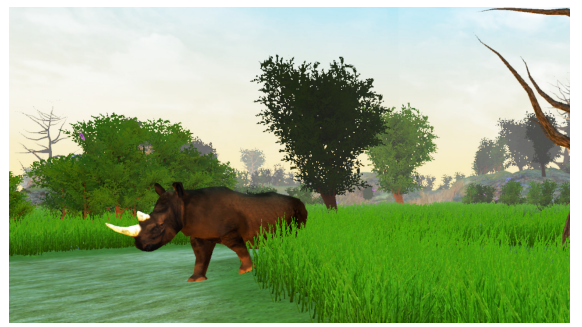

(d)

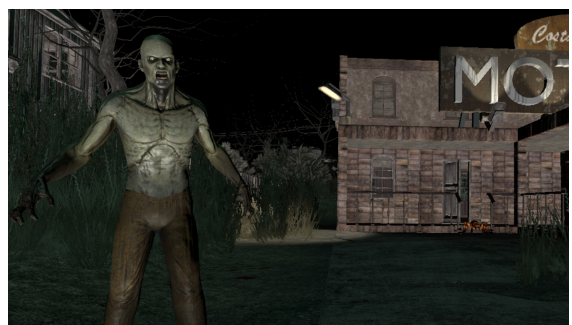

(b)

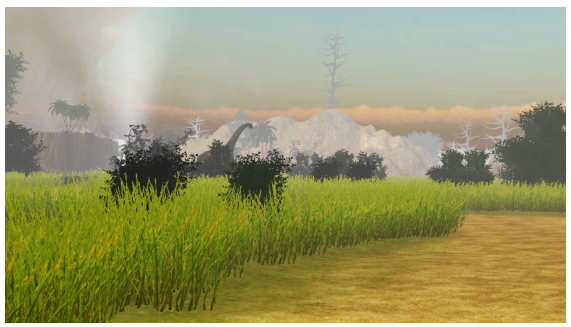

(e)

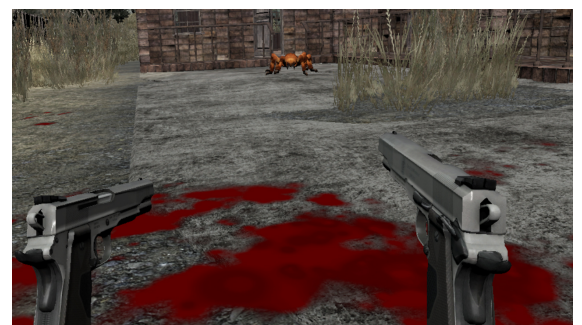

(c)

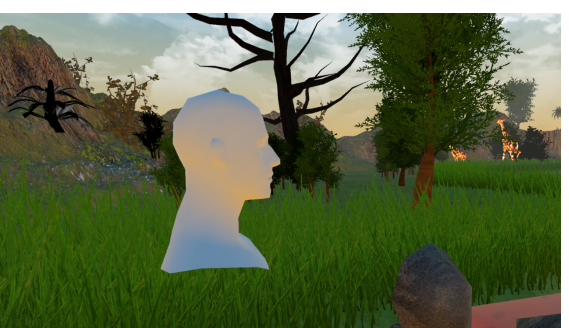

(f)

Figure 2: Representative scenes of the test environments. The active shoot to survive game (a-c) and passive jungle safari game (d-f). In both environments, the location and orientation of the collaborator was visualized by a unisex head model (f).

experimental tasks under six different conditions. We first welcomed the participants and explained the experiment. They then signed a consent form and wore the sensor on their torso underneath their clothes. Then we asked the participants to rest for two minutes and start filling out a demographic questionnaire. They also responded to the PANAS scale at the baseline. Following that they started performing the first experimental task. After each task they had to answer a set of questionnaires as mentioned in Section 4.2. It allowed participants to calm down from the earlier task. We also asked participants to rest for as long as they wanted before starting the next task. The process was repeated six times. One thing to note is that both participants in each pair responded to the same set of questions separately. In the end, we debriefed the participants and told them about the manipulation. Participants were allowed to leave the experiment anytime without giving us any reason. Overall, the experiment took almost two hours per pair on an average to complete.

\subsection{Hypotheses}

Before running the experiment we had a few hypotheses, as described below.

- H1: The active environment will cause the participant to have a higher heart rate than the passive environment, as this environment requires more interaction and physical movement.

- H2: Dey et al. [5] noticed that real feedback causes least intensity of five emotions in the PANAS scale - interested, excited, scared, nervous, and afraid. We expected the same emotions to be affected in our study as well.

- H3: As the higher heart rate perceptually relates to higher stress [15] and individuals under higher stress seek social presence [8], we expected in the higher manipulation levels participants will have a higher social presence.

- H4: For the same reason as H3, we also expected to have a higher IOS rating in $+20 \%$ manipulation level than the other two levels.

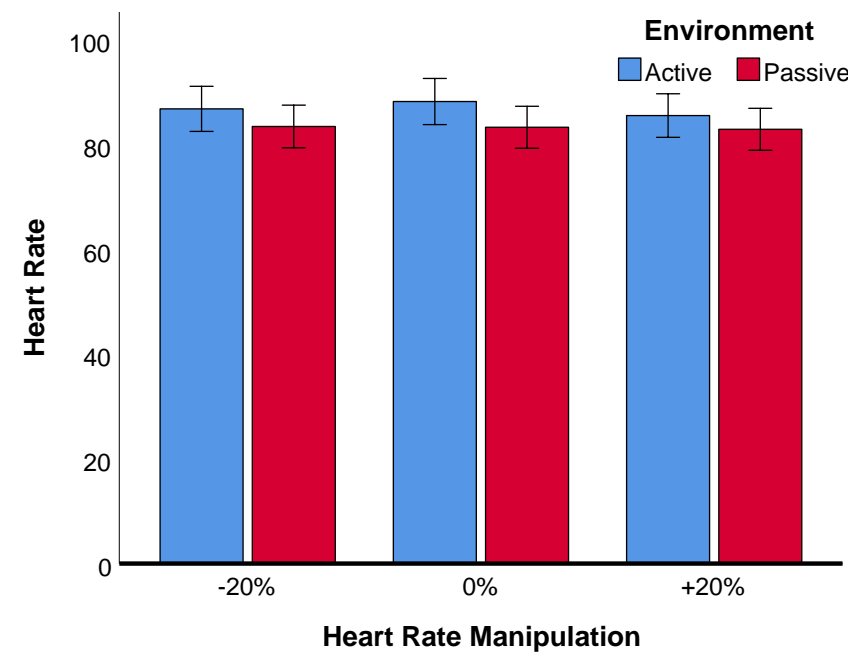

Figure 3: Heart rate during the experimental tasks. Whiskers represent $\pm 95 \%$ confidence interval.

- H5: We expected that participants will feel that they and their partners have the highest valance and arousal in the $+20 \%$ manipulation level.

\subsection{Participants}

We recruited 24 participants ( 5 females) in this study through social media advertisement, personal contacts, and university mailing lists. The participant ages ranged from between 19 and 46 years $(M=30.2$, $\mathrm{SD}=6.7)$. At the beginning we asked participants to rate their experience with VR out of ten. Our participant cohort was moderately experienced with VR $(\mathrm{M}=5.8, \mathrm{SD}=2.9)$. They reported doing work in collaboration with colleagues and friends in real life $(M=6.3$, $\mathrm{SD}=2.5)$. They moderately agreed to the statement "Someone's heart rate indicates that person's emotional state" $(\mathrm{M}=5.2, \mathrm{SD}=0.8)$. All of the participants had normal or corrected to normal vision. We 
created 12 pairs of participants to perform the experiment, with people in five pairs previously knowing each other. Prior to conducting the experiment we calculated the required sample size to achieve an acceptable power in the analysis using $\mathrm{G}^{*}$ Power version 3.1. We found that to achieve a power of $0.9,24$ participants were required. Hence, our sample size is sufficient for the experimental validity. Participation was voluntary and each of the participants were paid a $\$ 20$ gift voucher for their participation in the study.

Over the whole experiment, we had 24 (participants) $\times 3$ (manipulation levels $) \times 2$ (environment $)=144$ data points .

\section{Results}

\subsection{Heart Rate}

To analyze the effects of the independent variables on the participants' real heart rate we used a two-way repeated measure analysis of variance (ANOVA). We found a significant main effect of the environment $-\mathrm{F}(1,23)=7.1, p=.014, \eta_{p}^{2}=.24$, Observed Power $(\mathrm{OP})=.72$ (Figure 3 and Table A1). In the active environment heart rate was significantly higher than in the passive environment. We noticed a trend towards strong effect of the manipulation levels, although it was not statistically significant $-\mathrm{F}(2,46)=2.6, p=.08$, $\eta_{p}^{2}=.1$, Observed Power $(\mathrm{OP})=.49$. Interestingly, in both environments, $+20 \%$ manipulation had lowest real heart rate. There was no significant interaction effect.

\subsection{Self-Assessment Manikin (SAM)}

Data in the SAM questionnaire is non-parametric and ordinal, hence, we used the Wilcoxon Signed Ranks test for the Environment variable and Friedman's test for the manipulation variable. We noticed a significant effect of collaborative environments on valance $(\mathrm{Z}=-4.9, p<.001$, passive $>$ active $)$, arousal $(\mathrm{Z}=-5.1$, $p<.001$, passive $>$ active $)$, and dominance $(\mathrm{Z}=-5.1, p<.001$, passive $<$ active) when participants rated about their own experience. We noticed similar significant effects when participants rated about their partner-valance $(\mathrm{Z}=-3.3, \mathrm{p}=.001$, passive $>$ active $)$, arousal $(\mathrm{Z}=-3.3, \mathrm{p}=.001$, passive $>$ active $)$, and dominance $(\mathrm{Z}=-4.6$, $p<.001$, passive $<$ active). We also measured the difference in ratings between for themselves and for their partner. We only noticed a significant effect on valance $(\mathrm{Z}=-2.4, p=.015$, passive $>$ active $)$ meaning that in more cases of active environment than passive environment, participants rated their partner to have more positive emotions than themselves (Table A4).

For the manipulation levels, we did not notice any significant effect. However, there was trend towards significance in partner valance $\left(\chi^{2}(2)=4.7, \mathrm{p}=.09\right)$ and partner arousal $\left(\chi^{2}(2)=5.4, \mathrm{p}=.06\right)$ In case of partner valance (Figure $4 d$ ), participants perceived their partner to be the most positive emotionally in $+20 \%$ manipulation level followed by $0 \%$ and $-20 \%$. In the case of arousal (Figure $4 \mathrm{e}$ ), the effect reverses and participants perceived their partner to be most aroused in the $-20 \%$ manipulation level followed by $0 \%$ and $+20 \%$.

\subsection{Inclusion of The Other in Self Scale (IOS)}

As the data for the IOS scale is ordinal and non-parametric in nature we ran two separate Friedman's test for each of the independent variables. We found a significant effect of the environment on the IOS ratings $-\chi^{2}(1)=33.1, p<.001$ (see Figure 5 and Table A1). In the active shoot and survive environment participants felt more connected to each other than in the passive safari environment. However, we did not notice a significant effect of heart rate manipulation.

\subsection{Positive and Negative Affect Schedule (PANAS)}

With a set of repeated measure ANOVAs, we did not find a significant effect of either heart rate manipulations levels or environment on overall positive affect (PA) and negative affect (NA) as rated for self (Table A2) and the partner (Table A3). However, we found a significant correlation between PA and NA when reported for self- $\mathrm{PA}=13.23+1.15 \times \mathrm{NA}, p<.001$ and when reported for the partner- $\mathrm{PA}=16.86+.83 \times \mathrm{NA}, p<.001$. We also noticed that $\mathrm{PA}$ and NA correlated significantly for all levels of both independent variables, where PA was always more than NA.

Analysis of individual emotions and feelings

We then looked at the five individual emotions and feelingsinterested, excited, scared, nervous, and afraid. We separately looked at the data when reported for self and for partner.

Reported for self emotions and feeling

We noticed a significant interaction effect of heart rate manipulation $\times$ environment $-\mathrm{F}(2,46)=4.4, p=.02, \eta_{p}^{2}=.2, \mathrm{OP}=.73$ on the feeling of being scared (Figure 6a). A similar interaction effect was noticed for nervousness- $\mathrm{F}(2,46)=5.6, p<.01, \eta_{p}^{2}=.2, \mathrm{OP}=.83$ (Figure $6 \mathrm{~b}$ ). There were no other significant main and interaction effect found.

Reported for partner's perceived emotions and feelings

Participants perceived their partner to be significantly more excited in the active environment than in the passive one- $\mathrm{F}(1,23)=5, p=$ $.035, \eta_{p}^{2}=.2, \mathrm{OP}=.57$, which is consistent to our expectation (Figure $6 c)$. We did not find any other significant effect.

\subsection{Social Presence}

We used the Social Presence Questionnaire by Harms and Biocca [11], and the four sub-scales of the questionnaire which were relevant to our experiment-Co-presence, Attention allocation, Perceived message understanding, and Behavioral interdependence. Along with the sub-scales we have also calculated the total social presence as a sum of these sub-scales. To analyze the data we used two-way repeated measure ANOVAs.

In all cases, we have noticed a significant effect of collaborative environment on the Social Presence and in all scales active environment was rated better than the passive environment (see Figure 7 and Table A1). Total social presence- $\mathrm{F}(1,23)=44.18, p<.001, \eta_{p}^{2}=.66$, $\mathrm{OP}=1$; Co-presence- $\mathrm{F}(1,23)=44.18, p<.001, \eta_{p}^{2}=.66, \mathrm{OP}=1$; Attention allocation- $\mathrm{F}(1,23)=7.64, p=.01, \eta_{p}^{2}=.25$, OP=.75; Perceived message understanding $-\mathrm{F}(1,23)=30.36, p<.001, \eta_{p}^{2}=.57$, $\mathrm{OP}=1$; Behavioral interdependence- $\mathrm{F}(1,23)=47.72, p<.001$, $\eta_{p}^{2}=.66, \mathrm{OP}=1$. There were no other significant differences.

\subsection{Other Subjective Questions}

We asked the following six subjective questions, which participants answered on a five-point Likert scale with $1=$ Not at all and $5=$ Very, and a higher value indicated positive response except for the fifth question where the above scale was reversed.

1. How much attention did you pay to your partner's heart rate when in the game?

2. How much do you think that the heart rate visualization affected your experience when in the game?

3. How strongly did you feel the other person's heart rate during the task?

4. How much did you feel the other person's emotional state during the task?

5. How confused did you get with the heart rate feedback?

6. How much do you think your collaborator helped you in the task?

To analyze the subjective questions we used repeated measure ANOVAs (Figure 8). We did not notice any significant difference except for in Q3 and Q6. Q3 had a main effect of collaboration environments- $\mathrm{F}(1,23)=6.9, p=.015, \eta_{p}^{2}=.2, \mathrm{OP}=.71$, where passive environment $(\mathrm{M}=3.86, \mathrm{SD}=1.22)$ received higher ratings than the active environment $(\mathrm{M}=3.18, \mathrm{SD}=1.27)$, indicating that the participants felt the other collaborator's heart rate more in the passive 


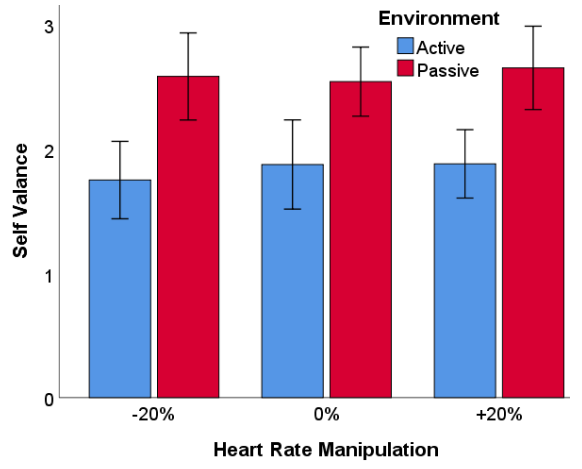

(a) Self Valance

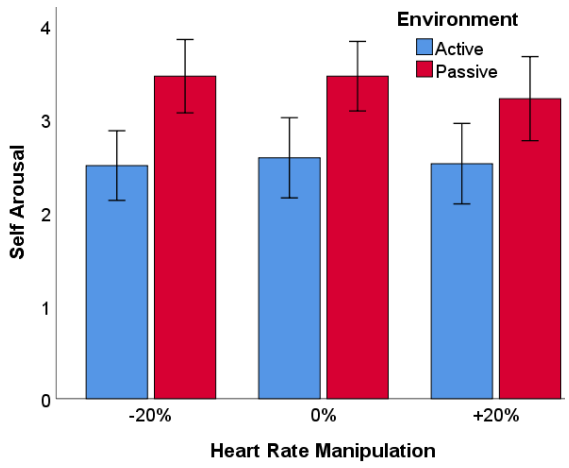

(b) Self Arousal

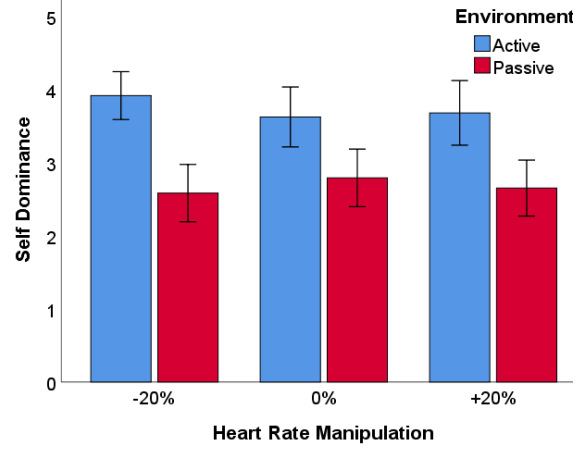

(c) Self Dominance

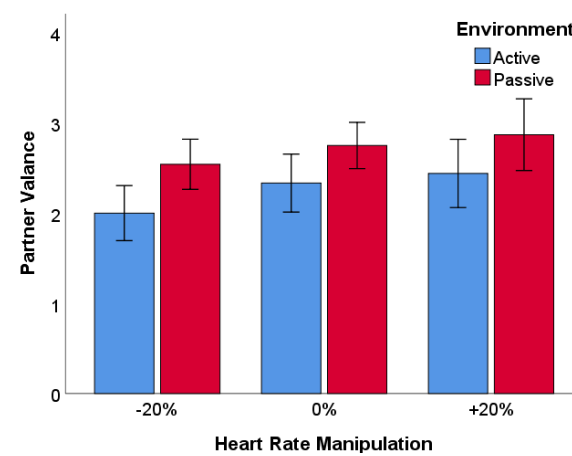

(d) Partner Valance

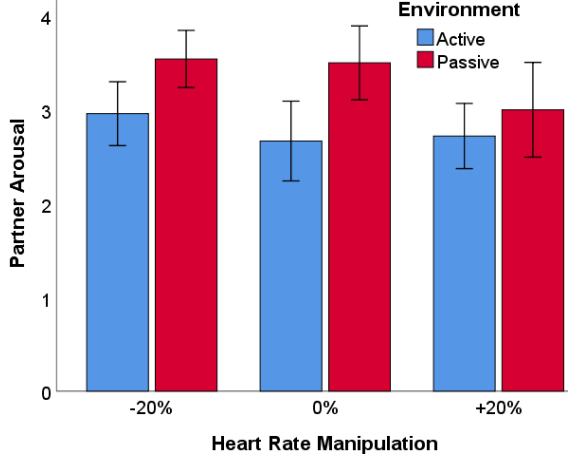

(e) Partner Arousal

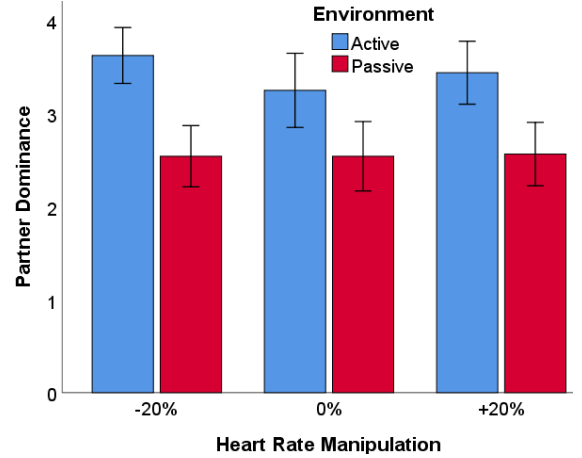

(f) Partner Dominance

Figure 4: Self Assessment Manikin ratings. Whiskers represent $\pm 95 \%$ confidence intervals.

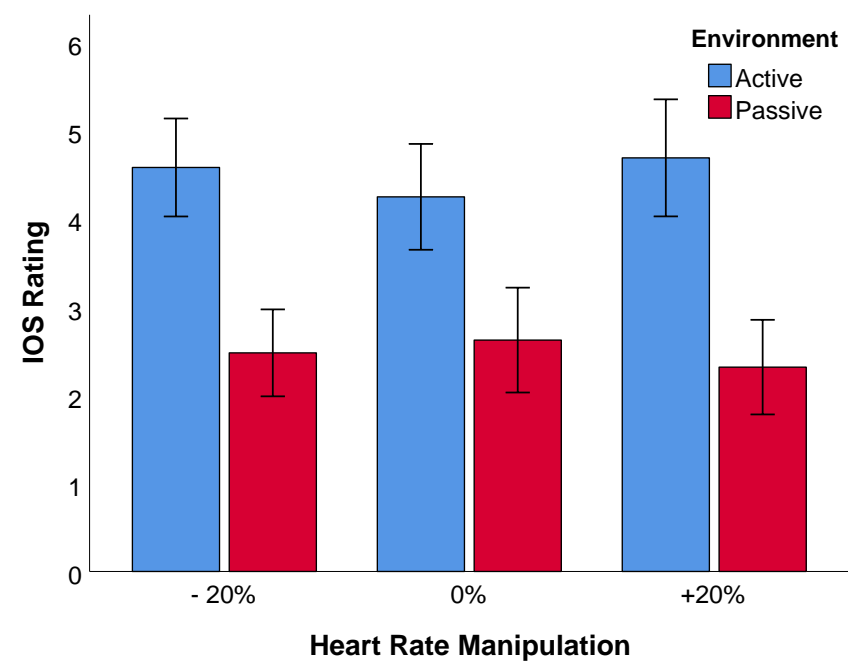

Figure 5: Inclusion of other in self scale. Whiskers represent $\pm 95 \%$ confidence interval.

environment than the active. Question Q6 had a main effect of collaborative environments $-\mathrm{F}(1,23)=87.7, p<.001, \eta_{p}^{2}=.8, \mathrm{OP}=1$, and the active environment $(\mathrm{M}=3.81, \mathrm{SD}=1.01)$ had higher ratings than the passive environment $(\mathrm{M}=1.73, \mathrm{SD}=0.97)$ indicating that participants felt that the other collaborator helped them more in the active environment than in the passive environment.

\section{Discussion}

In this section, we discuss the results in relation to the hypotheses. Our first hypothesis was The active environment will have higher heart rate than the passive environment as this environment requires more interaction and physical movement than the passive environment. Our analysis proved this hypothesis to be true. Our active environment required participants to shoot at the zombies and creatures and quickly look around to find them. We also noticed that participants communicated more with each other in this environment. These actions increased their heart rate. In contrast, the passive environment, did not require any bodily movement. Although, users were allowed to look around and communicate. However, they did not communicate as much in the passive environment. P12 mentioned "... the zombie game was more fun and I wanted to shoot at the zombies and to help my partner. But the safari was a calm experience, which I enjoyed but did not feel as pumpedt as in the zombie game." "

It was interesting to find that the physical heart rate was lower in the $+20 \%$ manipulation level in both environments. In our experiment participants experienced the heart rate of their collaborator. A higher heart rate provides a perception of an aroused state of the autonomic nervous system or a state that is commonly experienced while being under threat or feeling stressed [24]. This might have caused participants to feel that their partners were vulnerable and chances of their defeat is higher, which induced a social stress resulting in their own heart rate to be dropped [20].

Our second hypothesis, based on an earlier work by Dey et al. [5], stated that five relevant emotions in particular will be affected by the manipulated heart rate feedback-interested, excited, scared, nervous, and afraid. We asked the participants to rate their own emotions and that of their partner's using the PANAS scale. Our second 


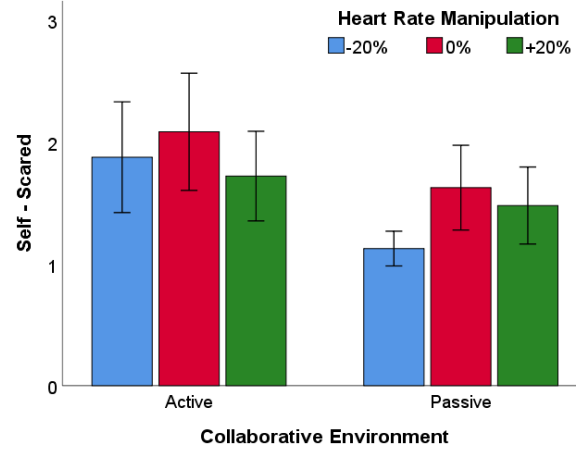

(a) Self Scared

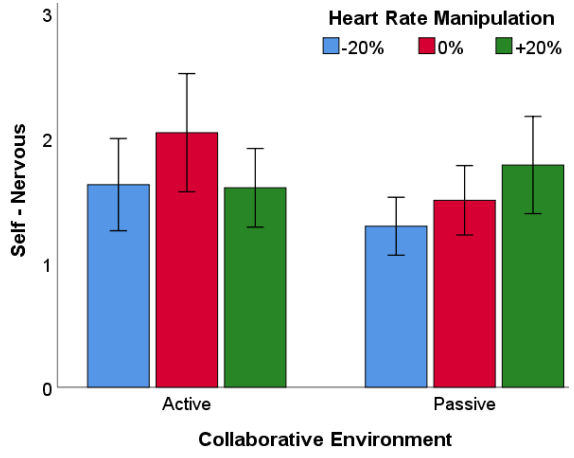

(b) Self Nervous

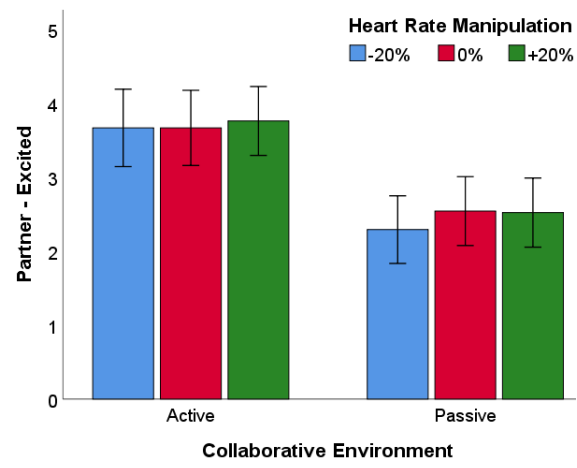

(c) Partner Excited

Figure 6: Positive and negative affect schedule ratings. Whiskers represent $\pm 95 \%$ confidence intervals.

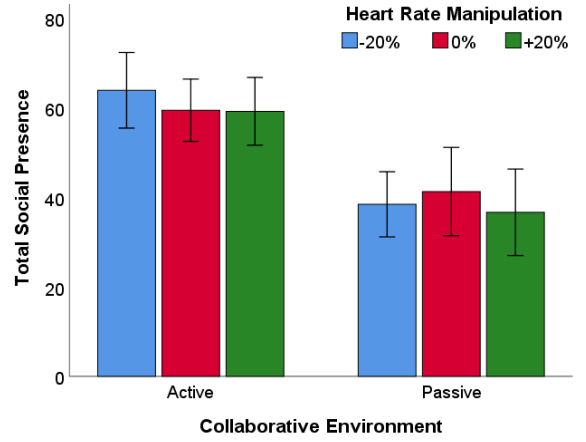

(a)

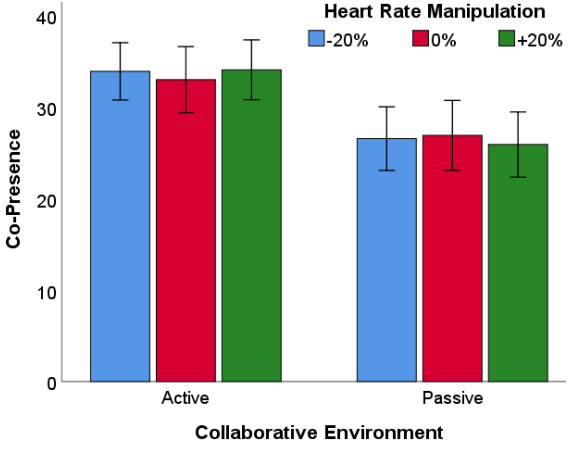

(b)

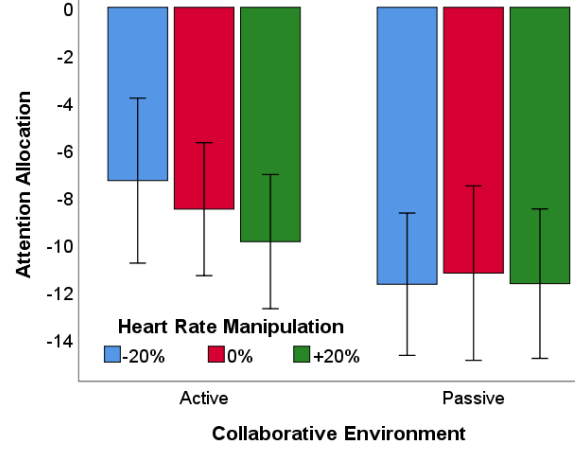

(c)

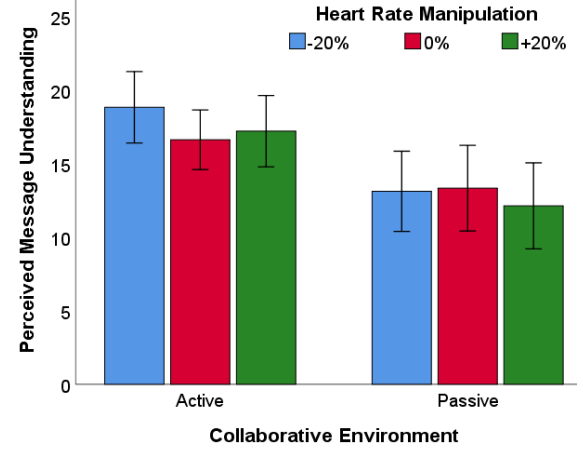

(d)

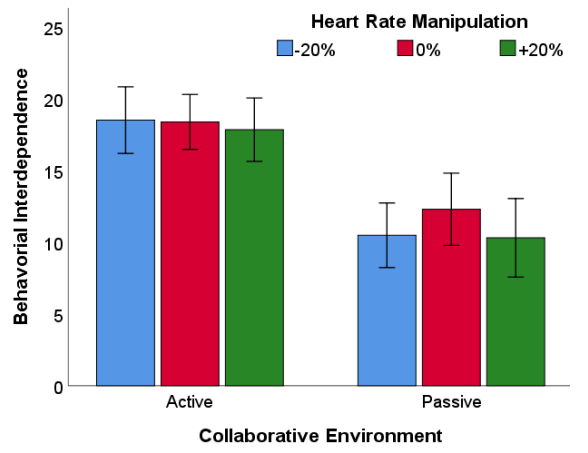

(e)

Figure 7: Ratings for social presence. Whiskers represent $\pm 95 \%$ confidence intervals.

hypothesis was partially accepted as there was an interaction effect between heart rate manipulation and the environments for the two emotions-scared and nervousness. For the feeling of being scared, we noticed a significant difference between the two environments for the $-20 \%$ manipulation level. However, for other manipulation levels the difference was not significant. We believe that the scary elements in the passive environment were not as profound as it was in the active environment. When participants noticed that their partner's heart rate was low, indicating that they are relaxed and in control of the experience, they themselves also felt relaxed and less scared. However, the same effect was not seen when they were in the active environment and shooting at the creatures to survive. This could be because they did not pay enough attention to the feedback. A few participants mentioned that they were more focused on the shooting task than the heartrate feedback. For example, P5 mentioned "... the shooting was fun and exciting but I am not sure whether I noticed the feedback as it was somehow hidden behind the sounds of gunshots."

This is an interesting finding as it identifies a need for more thoughtful feedback design based on the task at hand and the environmental elements such as sound and lighting. A salient visual feedback may be more appropriate than audio-haptic feedback in stressful and action-packed environments. We also noticed that the feeling of nervousness gradually increased with the increasing heartrate feedback in the passive environment but for the active environment $0 \%$ had the highest intensity of nervousness. This is possibly because in the passive environment participants had more opportunity to experience the feedback and get influenced by it than in the active environment where they needed to pay more attention 


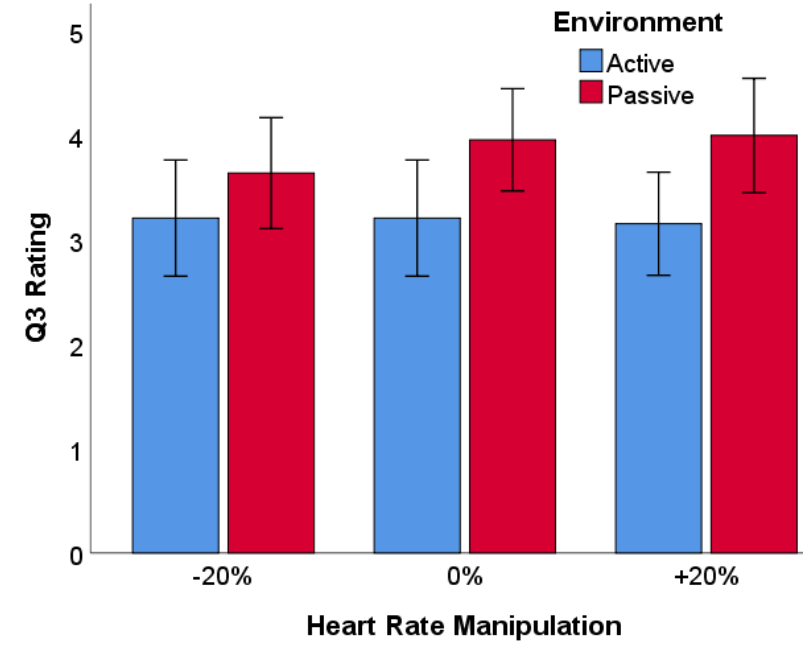

(a)

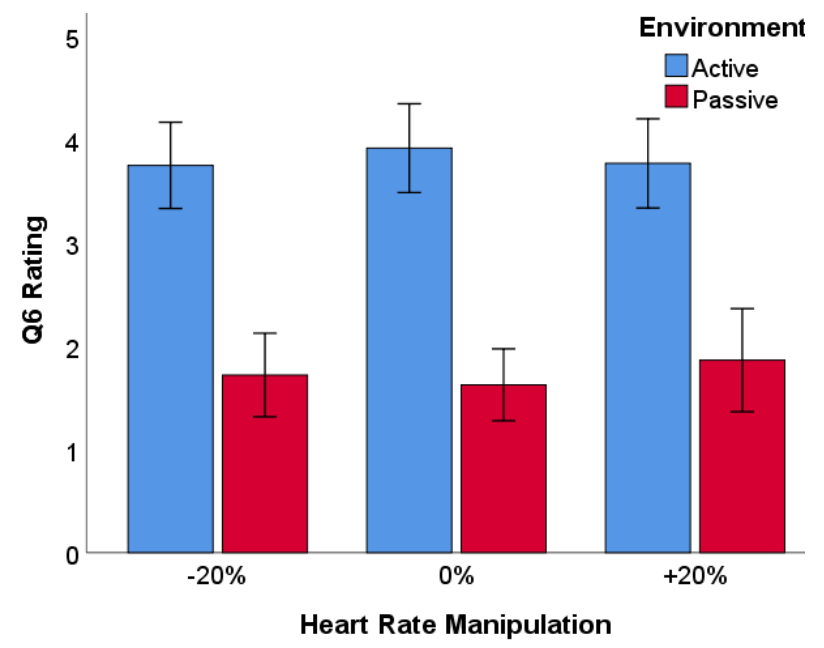

(b)

Figure 8: Ratings for the subjective questions 3 and 6 . Whiskers represent $\pm 95 \%$ confidence intervals.

to the task at hand.

In our third hypothesis, we expected that in the higher heart rate manipulation levels participants will have higher social presence. This hypothesis was rejected as we did not find a significant main effect of manipulation levels on overall Social Presence or on any of the sub-scales. We believe that this is because participants were not receiving their own heart rate feedback rather that of their collaborator. Based on previous findings $[8,15]$ we expected to see that a higher heart rate would induce higher Social Presence, however, the difference between earlier work and our work was that the feedback in our experiment was of a different individual and not that of the self. From our results, it is clear that a participant getting feedback from their own heart rate causes different effects than getting feedback of another person's heart rate. However, we noticed a significant main effect of environment where the active environment resulted in higher Social Presence than the passive environment. This is expected, as the active environment required more communication between the collaborators and surviving the zombie attacks by helping each other.

Our fourth hypothesis expected that higher heart rate manipulation levels will cause higher ratings in the IOS scale. This hypothesis was not supported as there was no significant effect of heart rate manipulation levels on the IOS ratings. However, we noticed that active environment caused significantly higher IOS ratings than the passive environment. We believe as the task in the active environment required more communication and collaboration to survive, causing participants to feel a closer connection with the collaborator.

Our fifth hypothesis expected the $+20 \%$ manipulation level will cause highest valance and arousal for both the self and partner. This hypothesis was not supported at the significance levels tested. However, we did notice a strong trend towards significance when rated for partner valence $(\mathrm{p}=.09)$ and arousal $(\mathrm{p}=.06)$. For valance, participants perceived their partner to have positive emotion most in the $+20 \%$ manipulation level. This could be because in the high heart rate feedback levels participants perceived their partner to be excited and enjoying the experience. P14 explained "... I think he (the partner) was having a great time as his heart rate was going up as he was shooting." For arousal, however, the effect was revered and participants perceived their partner to have the highest arousal in the $-20 \%$ manipulation level. The reason for this counter-intuitive effect is unclear and requires further experimentation. Otherwise, for the SAM questionnaire, we noticed that participants thought that they and their partners were more aroused and had higher valance in the active environment than in the passive environment. This is expected, and consistent to earlier findings, as more interaction with the environment in VR increases engagement and Presence [14]. Most of the participants in our experiment complained about not being able to interact with the animals and the trees in the safari.

Overall, out of the five hypotheses two were either fully or partially accepted. While we have noticed a strong effect of virtual environments to cause differences emotionally and physiologically, the direct impact of heart rate manipulation was subtle but noticeable in some situations such as partner valance and arousal. Participants liked the idea of being able to understand the other person's physiological state during collaboration but in the given tasks in this experiment they were not always effective. One participant (P3) said that "... it is interesting to know the other person's heart rate but I do not think it changed my actions at all in the given circumstances."

\section{Conclusion and Future Work}

In this paper, we have presented the first study where a collaborator's heart rate feedback was shared with another collaborator in a virtual environment in a modified form. We used two different virtual environments with two different levels of interaction-passive (safari) and active (shoot and survive). In each of those environments we provided three levels of manipulated heart rate feedback $(-20 \%$, $0 \%$, and $+20 \%$ ) of the collaborators to each other in real time. We explored the effects of such manipulations and the different VEs in terms of real heart rate, emotional effects, and social presence.

We have found that nervousness and scariness in the VEs can be manipulated by providing manipulated heart rate feedback of one collaborator to the other. Manipulation of heart rate feedback affects the perceived valance and arousal levels of one another during collaboration. Our active VEs caused higher Social Presence, IOS, and PANAS than passive VEs. Overall, the utility of providing heart rate feedback to the other collaborator is depended on the task at hand. We suggest providing increased heart rate feedback where it is important to make the collaborators feel that the other collaborator is in a positive emotional state (valance). Increased heart rate feedback enhance the feeling of nervousness in a passive environment. We suggest that positively manipulated heart rate feedback be provided in less active VEs where increasing nervousness may be useful such as in VR movies. 


\subsection{Limitations}

We have presented the first experiment where the effects of sharing manipulated heart rate feedback of one collaborator to the other is measured but our experiment has a few limitations. First, our experimental environments were entertaining in nature and not relevant to real world experiences. Our results, accordingly, may yield differences in a different set of tasks where the experiences are more serious in nature such as remote training and tele-medicine.

Second, informed by [5], we have manipulated the heart rate signals within $\pm 20 \%$ range. While these manipulation levels have indicated some effects in the measured variables, wider range of manipulation (e.g. $\pm 50 \%$ ) might have resulted in stronger differences.

Third, we besides heart rate there are other physiological signals that corresponds to human emotions such has galvanic skin response (GSR) and pupil dilation [3]. In our experiment we have not measured or shared these signals. While, we have noticed a trend of heart rate feedback manipulation resulting in a change in real heart rate, we did not measure what effects it had on other physiological signals.

\subsection{Future Work}

In the future, we will explore more serious collaborative environments such as training and surgery where heart rate feedback may provide more utility than the entertaining VEs used in this experiment, especially for conveying information about stress levels. The method of providing the feedback is another area to explore further As we noticed in the active environment, participants did not notice the feedback as much, so it will be interesting to explore how the feedback can be made more salient in these kinds of environments without compromising the experience. In this study, we have only used heart rate for measuring and providing feedback. It will also be interesting to investigate providing feedback for other physiological and neurological signals such as galvanic skin response, respiration, and alpha waves.

\section{References}

[1] G. Bernal and P. Maes. Emotional beasts: Visually expressing emotions through avatars in vr. In Proceedings of the 2017 CHI Conference Extended Abstracts on Human Factors in Computing Systems, pp. 2395-2402. ACM, 2017.

[2] M. M. Bradley and P. J. Lang. Measuring emotion: the self-assessment manikin and the semantic differential. Journal of behavior therapy and experimental psychiatry, 25(1):49-59, 1994.

[3] H. Chen, A. Dey, M. Billinghurst, and R. W. Lindeman. Exploring pupil dilation in emotional virtual reality environments. In Proceedings of the International Conference on Artificial Reality and Telexistence Eurographics Symposium on Virtual Environments, pp. 1-8, 2017.

[4] H. Chen, A. Dey, M. Billinghurst, and R. W. Lindeman. Exploring the design space for multi-sensory heart rate feedback in immersive virtual reality. In Proceedings of the 29th Australian Conference on Computer-Human Interaction, pp. 108-116. ACM, 2017.

[5] A. Dey, H. Chen, M. Billinghurst, and R. W. Lindeman. Effects of manipulating physiological feedback in immersive virtual environments. In Proceedings of the 2018 Annual Symposium on Computer-Human Interaction in Play, pp. 101-111. ACM, 2018.

[6] A. Dey, H. Chen, C. Zhuang, M. Billinghurst, and R. W. Lindeman. Effects of sharing real-time multi-sensory heart rate feedback in different immersive collaborative virtual environments. In Proceedings of the IEEE International Symposium on Mixed and Augmented Reality. IEEE, 2018.

[7] A. Dey, T. Piumsomboon, Y. Lee, and M. Billinghurst. Effects of sharing physiological states of players in a collaborative virtual reality gameplay. In Proceedings of the 2017 CHI Conference on Human Factors in Computing Systems, pp. 4045-4056. ACM, 2017.

[8] A. Felnhofer, H. Hlavacs, L. Beutl, I. Kryspin-Exner, and O. D. Kothgassner. Physical presence, social presence, and anxiety in participants with social anxiety disorder during virtual cue exposure. Cyberpsychology, Behavior, and Social Networking, 22(1):46-50, 2018.
[9] A. Felnhofer, O. D. Kothgassner, M. Schmidt, A.-K. Heinzle, L. Beutl, H. Hlavacs, and I. Kryspin-Exner. Is virtual reality emotionally arousing? investigating five emotion inducing virtual park scenarios. International journal of human-computer studies, 82:48-56, 2015.

[10] H. Fukushima, Y. Terasawa, and S. Umeda. Association between interoception and empathy: evidence from heartbeat-evoked brain potential. International Journal of Psychophysiology, 79(2):259-265, 2011.

[11] C. Harms and F. Biocca. Internal consistency and reliability of the networked minds measure of social presence. In Proceedings of the Seventh Annual International Workshop: Presence 2004, 2004.

[12] M. Hassib, D. Buschek, P. W. Wozniak, and F. Alt. Heartchat: Heart rate augmented mobile chat to support empathy and awareness. In Proceedings of the 2017 CHI Conference on Human Factors in Computing Systems, CHI '17, pp. 2239-2251. ACM, New York, NY, USA, 2017. doi: $10.1145 / 3025453.3025758$

[13] M. Meehan, B. Insko, M. Whitton, and F. P. Brooks Jr. Physiological measures of presence in stressful virtual environments. Acm transactions on graphics (tog), 21(3):645-652, 2002.

[14] H. Regenbrecht and T. Schubert. Real and illusory interactions enhance presence in virtual environments. Presence: Teleoperators $\mathcal{E}$ Virtual Environments, 11(4):425-434, 2002.

[15] T. Reinhardt, C. Schmahl, S. WÃijst, and M. Bohus. Salivary cortisol, heart rate, electrodermal activity and subjective stress responses to the mannheim multicomponent stress test (mmst). Psychiatry Research, 198(1): 106 - 111, 2012. doi: 10.1016/j.psychres.2011.12.009

[16] G. Riva, F. Mantovani, C. S. Capideville, A. Preziosa, F. Morganti, D. Villani, A. Gaggioli, C. Botella, and M. Alcañiz. Affective interactions using virtual reality: the link between presence and emotions. CyberPsychology $\mathcal{E}$ Behavior, 10(1):45-56, 2007.

[17] A. Rizzo, J. F. Morie, J. Williams, J. Pair, and J. G. Buckwalter. Human emotional state and its relevance for military vr training. Technical report, University Of Southern California Marina Del Rey Ca Inst For Creative Technologies, 2005.

[18] M. J. Roy, M. E. Costanzo, T. Jovanovic, S. Leaman, P. Taylor, S. D. Norrholm, and A. A. Rizzo. Heart rate response to fear conditioning and virtual reality in subthreshold ptsd. Annual Review of Cybertherapy and Telemedicine, 191:115-119, 2013.

[19] S. Seinfeld, I. Bergstrom, A. Pomes, J. Arroyo-Palacios, F. Vico, M. Slater, and M. V. Sanchez-Vives. Influence of music on anxiety induced by fear of heights in virtual reality. Frontiers in psychology, 6:1969, 2016.

[20] A. Sgoifo, J. M. Koolhaas, E. Musso, and S. F. De Boer. Different sympathovagal modulation of heart rate during social and nonsocial stress episodes in wild-type rats. Physiology Behavior, 67(5):733-738, 1999.

[21] P. Shah, C. Catmur, and G. Bird. From heart to mind: linking interoception, emotion, and theory of mind. Cortex; a journal devoted to the study of the nervous system and behavior, 93:220, 2017.

[22] P. Slovák, J. Janssen, and G. Fitzpatrick. Understanding heart rate sharing: Towards unpacking physiosocial space. In Proceedings of the SIGCHI Conference on Human Factors in Computing Systems, CHI '12, pp. 859-868. ACM, New York, NY, USA, 2012. doi: 10. $1145 / 2207676.2208526$

[23] C. S. S. Tan, J. Schöning, K. Luyten, and K. Coninx. Investigating the effects of using biofeedback as visual stress indicator during videomediated collaboration. In Proceedings of the SIGCHI Conference on Human Factors in Computing Systems, pp. 71-80. ACM, 2014.

[24] T. D. Wager, C. E. Waugh, M. Lindquist, D. C. Noll, B. L. Fredrickson, and S. F. Taylor. Brain mediators of cardiovascular responses to social threat: Part i: Reciprocal dorsal and ventral sub-regions of the medial prefrontal cortex and heart-rate reactivity. NeuroImage, 47(3):821 835, 2009. Brain Body Medicine. doi: 10.1016/j.neuroimage.2009.05. 043

[25] D. Watson, L. A. Clark, and A. Tellegen. Development and validation of brief measures of positive and negative affect: the panas scales. Journal of personality and social psychology, 54(6):1063, 1988.

[26] B. K. Wiederhold, D. P. Jang, S. I. Kim, and M. D. Wiederhold. Physiological monitoring as an objective tool in virtual reality therapy. CyberPsychology $\mathcal{E}$ Behavior, 5(1):77-82, 2002. 


\begin{tabular}{llrrrrrrr}
\hline \multirow{2}{*}{ Manipulation } & Environment & \multicolumn{9}{c}{ Social Presence } & IOS & Heart Rate \\
\cline { 3 - 6 } & & \multicolumn{1}{c}{ CP } & \multicolumn{1}{c}{ AA } & \multicolumn{1}{c}{ PMU } & BI & \multicolumn{1}{c}{ Overall } & & \\
\hline$-20 \%$ & Active & $33.9(7.4)$ & $-7.3(8.2)$ & $18.8(5.7)$ & $18.5(5.4)$ & $63.9(20)$ & $4.6(1.3)$ & $86.8(10)$ \\
& Passive & $26.5(8.4)$ & $-11.7(7.2)$ & $13.1(6.6)$ & $10.4(5.4)$ & $38.4(17.6)$ & $2.5(1.2)$ & $83.4(9.4)$ \\
$0 \%$ & Active & $33(8.5)$ & $-8.5(6.6)$ & $16.6(4.7)$ & $18.3(4.5)$ & $59.4(16.4)$ & $4.3(1.4)$ & $88.2(10.2)$ \\
& Passive & $26.9(9)$ & $-11.2(8.7)$ & $13.3(6.9)$ & $12.2(5.9)$ & $41.2(23.3)$ & $2.6(1.4)$ & $83.3(9.3)$ \\
$20 \%$ & Active & $34(7.7)$ & $-9.9(6.7)$ & $17.2(5.7)$ & $17.8(5.2)$ & $59.2(17.9)$ & $4.7(1.6)$ & $85.5(9.6)$ \\
& Passive & $25.9(8.2)$ & $-11.6(7.3)$ & $12.1(6.7)$ & $10.3(6.3)$ & $36.6(22.3)$ & $2.3(1.2)$ & $82.9(9.3)$ \\
\hline
\end{tabular}

Table A1: Mean and standard deviation values of social presence, inclusion of other in self scale (IOS), and heart rate. $\mathrm{CP}=\mathrm{Co}-\mathrm{presence}, \mathrm{AA}$ $=$ Attention Allocation, PMU = Perceived message understanding, $\mathrm{BI}=$ Behavioral interdependence.

\begin{tabular}{|c|c|c|c|c|c|c|c|c|}
\hline \multirow{2}{*}{\multicolumn{2}{|c|}{ Manipulation Environment }} & \multicolumn{7}{|c|}{ PANAS (Self) } \\
\hline & & Interested & Excited & Scared & Nervous & Afraid & PA & NA \\
\hline \multirow[t]{2}{*}{$-20 \%$} & Active & $4.2(1.1)$ & $3.7(1.3)$ & $1.8(1)$ & $1.6(0.8)$ & $1.5(0.9)$ & $36(10.8)$ & $15.8(6.1)$ \\
\hline & Passive & $3(1.2)$ & $2.5(1.2)$ & $1.1(0.3)$ & $1.2(0.5)$ & $1.1(0.3)$ & $23.8(10.2)$ & $12(3.3)$ \\
\hline \multirow[t]{2}{*}{$0 \%$} & Active & $4.1(1)$ & $3.7(1.2)$ & $2(1.1)$ & $2(1.1)$ & $1.7(1.2)$ & $36(10.6)$ & $16.8(6.8)$ \\
\hline & Passive & $3.1(1.2)$ & $2.8(1.3)$ & $1.6(0.8)$ & $1.5(0.6)$ & $1.3(0.6)$ & $25.8(9.6)$ & $14.1(4.9)$ \\
\hline \multirow[t]{2}{*}{$20 \%$} & Active & $4.2(1)$ & $3.8(1.3)$ & $1.7(0.8)$ & $1.6(0.7)$ & $1.5(1)$ & $35.8(11.1)$ & $15.7(5.9)$ \\
\hline & Passive & $2.9(1.4)$ & $2.6(1.1)$ & $1.4(0.7)$ & $1.7(0.9)$ & $1.4(0.7)$ & $24(9.1)$ & $14.34(4.8)$ \\
\hline
\end{tabular}

Table A2: Mean and standard deviation values of Positive and Negative Affect Schedule (PANAS) ratings when rated for self. PA = Positive Affect, NA = Negative Affect.

\begin{tabular}{llrrrrrrr}
\hline \multirow{2}{*}{ Manipulation Environment } & \multicolumn{7}{c}{ PANAS (Partner) } \\
\cline { 2 - 8 } & & Interested & Excited & Scared & Nervous & \multicolumn{1}{c}{ Afraid } & \multicolumn{1}{c}{ PA } & \multicolumn{1}{c}{ NA } \\
\hline$-20 \%$ & Active & $1.7(1)$ & $4(1.1)$ & $3.6(1.2)$ & $1.4(0.7)$ & $1.3(0.7)$ & $34.5(9.9)$ & $14.7(5.2)$ \\
& Passive & $1.2(0.6)$ & $2.9(1.1)$ & $2.2(1)$ & $1.3(0.6)$ & $1.1(0.3)$ & $22.8(9)$ & $11.8(2.9)$ \\
$0 \%$ & Active & $2.1(1.2)$ & $3.9(1)$ & $3.6(1.2)$ & $1.8(0.9)$ & $1.8(1.2)$ & $33.7(10.3)$ & $17(8.1)$ \\
& Passive & $1.3(0.7)$ & $3.1(1.2)$ & $2.5(1.1)$ & $1.3(0.6)$ & $1.3(0.7)$ & $24.4(8.9)$ & $13.2(4.8)$ \\
$20 \%$ & Active & $1.7(0.9)$ & $4(1)$ & $3.7(1.1)$ & $1.7(0.8)$ & $1.4(1)$ & $34(10.2)$ & $15.5(5.6)$ \\
& Passive & $1.5(0.8)$ & $2.9(1.3)$ & $2.5(1)$ & $2(1)$ & $1.5(0.8)$ & $23.3(8.5)$ & $14.6(6)$ \\
\hline
\end{tabular}

Table A3: Mean and standard deviation values of Positive and Negative Affect Schedule (PANAS) ratings when rated for partner. PA = Positive Affect, NA = Negative Affect.

\begin{tabular}{lllllllll}
\hline \multirow{2}{*}{ Manipulation Environment } & \multicolumn{3}{c}{ SAM (Self) } & & \multicolumn{3}{c}{ SAM (Partner) } \\
\cline { 3 - 4 } \cline { 8 - 9 } & & Valance & Arousal & Dominance & & Valance & Arousal & Dominance \\
\hline$-20 \%$ & Active & $1.7(0.7)$ & $2.5(0.8)$ & $3.9(0.7)$ & & $2(0.7)$ & $2.9(0.8)$ & $3.6(0.7)$ \\
& Passive & $2.5(0.8)$ & $3.4(0.9)$ & $2.5(0.9)$ & & $2.5(0.6)$ & $3.5(0.7)$ & $2.5(0.7)$ \\
$0 \%$ & Active & $1.8(0.8)$ & $2.5(1)$ & $3.6(0.9)$ & & $2.3(0.7)$ & $2.6(1)$ & $3.2(0.9)$ \\
& Passive & $2.5(0.6)$ & $3.4(0.8)$ & $2.7(0.9)$ & & $2.7(0.6)$ & $3.5(0.9)$ & $2.5(0.8)$ \\
$20 \%$ & Active & $1.8(0.6)$ & $2.5(1)$ & $3.6(1)$ & $2.4(0.9)$ & $2.7(0.8)$ & $3.4(0.8)$ \\
& Passive & $2.6(0.7)$ & $3.2(1)$ & $2.6(0.8)$ & $2.8(0.9)$ & $3(1.1)$ & $2.5(0.7)$ \\
\hline
\end{tabular}

Table A4: Mean and standard deviation values of Self Assessment Manikin (SAM) scale ratings for self and partner. 